\title{
A TABU SEARCH APPROACH FOR THE RECONSTRUCTION OF BINARY IMAGES WITHOUT EMPTY INTERIOR REGION
}

\author{
ALAIN BILLIONNET, FETHI JARRAY, GHASSEN TLIG AND EZZEDINE ZAGROUBA
}

\begin{abstract}
In this paper, we are concerned with a discrete tomography problem. We seek to reconstruct a binary image from its orthogonal projections, i.e, its horizontal and vertical line sums without interior black holes. We provide a tabu search approach to minimize the number of holes while satisfying the projections. We test our approach on some random binary images. Computational results show that the algorithm proposed produces near-optimal solutions for all test problems.
\end{abstract}

\section{INTRODUCTION}

Discrete tomography is a modern inverse problem in which we consider the horizontal and the vertical line sums of an image as a basis to obtain the pixels of the image. Binary images are most commonly represented by binary matrices with values 1 (white pixel) and 0 (black pixel). We will refer to any pixel of the image by its matrix position (see Fig. 1).

Discrete tomography is applicable in many interesting contexts to reconstruct discrete structures such as Workforce Scheduling $[9,16]$, Data Compression and Data Security [15], Industrial Non-Destructive Testing [4], Medical Imaging [13,17] and Timetabling [5].

In a binary image, we call the horizontal projection of row $i$, the number of white pixels in this row. Similarly, we call the vertical projection of column $j$, the number of white pixels in column $j$. The basic problem of reconstructing a binary matrix from its orthogonal projections is defined as follows: given the horizontal projection of each row and the vertical projection of each column, find a binary image that fits with the prescribed projections. Ryser described [18] a polynomial time algorithm to solve such a problem and gave necessary and sufficient conditions on the projections for the existence of a reconstruction.

Generally, the basic problem is underdetermined and many solutions may exist. To reduce the space of feasible solutions, various forms of prior knowledge on size, shape, smoothness, etc. are integrated to uniquely reconstruct the original structure from the projections. Incorporating such information into a method is a difficult task and the reconstruction problem is usually reduced to an optimization problem to select the best solution in a certain sense. In the literature, several additional constraints and prior knowledge have been considered to reduce the set

$M S C$ (2010): primary 34K06, 34K25.

Keywords: discrete tomography, tabu search, adjacency binary images, interior holes binary images. 
of the feasible solutions of the basic problem. These constraints include periodicity $[10,11]$, convexity $[1-3,6,8,14]$, adjacency [5], connectedness $[3,7,12,20]$ and timetabling [5].

In this paper, we deal with the reconstruction of binary images without interior black holes. A hole can be considered as an individual 0 (black pixels) surrounded by $1 \mathrm{~s}$ (white pixels). In particular, our research objective is to develop a tabu search approach to reconstruct a binary image with a minimal number of black holes. As far as the authors know, no one has so far published research on the reconstruction of such images.

The remainder of this paper is organized as follows. In Section 2, we introduce some definitions and notation. In Section 3, we propose a tabu search approach. In Section 4, we present and discuss the numerical results.

\section{Preliminaries}

Throughout this paper, we assume that the binary images are of size $m \times n$. Given an $m \times n$ binary image $A=\left[a_{i, j}\right]$, the horizontal projection of $A$ is the vector $H=\left(h_{1}, \ldots, h_{m}\right)$ such that $h_{i}=\sum_{j=1}^{n} a_{i, j}$ is the number of white cells on row $i$. The vertical projection of $A$ is defined analogously as the vector $V=\left(v_{1}, \ldots, v_{n}\right)$ where $v_{j}=\sum_{i=1}^{m} a_{i, j}$ is the number of white cells on column $j$. Both projections $H$ and $V$ constitute the orthogonal projections of $A$ (see Fig. 1). We denote by $B M(H, V)$ the class of all binary images satisfying the orthogonal projections $H$ and $V$. The condition $\sum_{i=1}^{m} h_{i}=\sum_{j=1}^{n} v_{j}\left(C_{0}\right)$ is obviously necessary for the existence of a binary image satisfying both projections.

\begin{tabular}{|c|c|c|c|c|c|c|c|c|}
\hline 0 & 1 & 0 & 0 & 1 & 1 & 0 & 1 & 0 \\
\hline 1 & 1 & 0 & 1 & 1 & 1 & 0 & 1 & 0 \\
\hline 1 & 1 & 1 & 0 & 0 & 0 & 1 & 0 & 0 \\
\hline 0 & 1 & 0 & 1 & 0 & 0 & 1 & 0 & 1 \\
\hline 0 & 1 & 0 & 1 & 1 & 0 & 0 & 1 & \\
\hline
\end{tabular}

Figure 1. A binary image with horizontal projection $H=(5,3,5,6,4)$ and vertical projection $V=(2,1,3,3,3,2,2,3,4)$.

The reconstruction of a black and white image from horizontal and vertical line sums is stated as follows:

Reconstruction Binary Image: $R B I(H, V)$

Given: $H=\left(h_{1}, \ldots, h_{m}\right) \in \mathbb{N}^{m}$ and $V=\left(v_{1}, \ldots, v_{n}\right) \in \mathbb{N}^{n}$.

Goal: Construct an $m \times n$ binary image that satisfies $H$ and $V$, i.e., row $i$ has exactly $h_{i}$ black cells and column $j$ has exactly $v_{i}$ black cells. 
The number of binary images in the class $B M(H, V)$ is very large [19]. The definition of switching components is an essential concept to describe the characteristic of the class $B M(H, V)$.

Definition 2.1. A switching component of a binary image is a $2 \times 2$ sub image of the form $\left(\begin{array}{ll}1 & 0 \\ 0 & 1\end{array}\right)$ or $\left(\begin{array}{ll}0 & 1 \\ 1 & 0\end{array}\right)$. A switching operation consists in interchanging the 0's and 1's of a switching component.

We note that a switching operation preserves the orthogonal projections of images. The following theorem is the starting point for the meta-heuristics of the reconstruction of binary images under various constraints [18] since it shows that the graph of the neighborhood is connected.

Theorem 2.2. [18] Let $A$ and $B$ in $B M(H, V), B \neq A$. Then, $A$ is transformable into $B$ (or vice versa) by finite sequences of switching operations.

Definition 2.3. For a binary image a cell $(i, j)$ is a hole if $(i, j)$ is a black pixel surrounded by 4 white pixels.

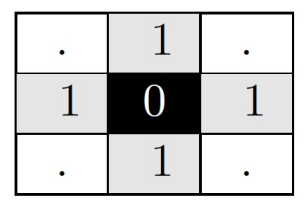

Figure 2. Illustration of a black hole pixel.

We say that a binary image fulfills the non-hole constraint if none of its black pixels is a hole. We denote $R N H(H, V)$ the problem of reconstructing an $m \times n$ binary image without hole pixels from its horizontal $H=\left(h_{1}, \ldots, h_{m}\right)$ and vertical $V=\left(v_{1}, \ldots, v_{n}\right)$ projections. The problem $R N H(H, V)$ can be defined as follows:

Reconstruction of Non-Hole Binary Image: $R N H(H, V)$

Given: $H=\left(h_{1}, \ldots, h_{m}\right) \in \mathbb{N}^{m}$ and $V=\left(v_{1}, \ldots, v_{n}\right) \in \mathbb{N}^{n}$.

Goal: Construct an $m \times n$ binary image without hole that satisfies the row and column sums $H$ and $V$.

In this paper, we are mainly interested in the problem $R N H(H, V)$, i.e., we consider the four neighbors of each pixel.

\section{TABU SEARCH APPROACH OF NON INTERIOR HOLE BINARY IMAGES}

We develop a tabu search algorithm for approximating binary images without interior holes.

\subsection{Initial solution}

We use Ryser's classical algorithm $O(m n+\max (m \operatorname{logn}, n \log m))$ to reconstruct an initial solution satisfying the orthogonal projections. This polynomial time algorithm can be described as follows: 


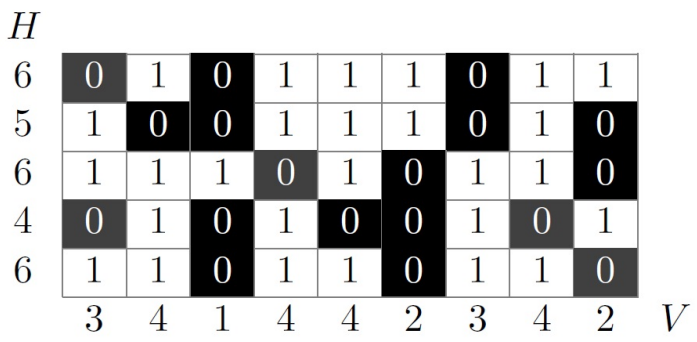

Figure 3. An example of binary image with 5 black holes.

\section{Ryser's algorithm}

Input: $\left\{H=\left(h_{1}, \ldots, h_{m}\right), V=\left(v_{1}, \ldots, v_{n}\right)\right.$ two nonnegative integer vectors $\}$.

Output: $\{A \in B M(H, V)\}$.

For $\mathrm{i}=1$ to $\mathrm{n}$ do

If (the number of available rows $\geq v_{j}$ ) then

Set $v_{j}$ ones in priorities row;

For each one placed in row i do: $h_{i} \longleftarrow h_{i}-1$;

Else no solution;

\section{End For}

In each step, row 'i' is called available if $h_{i}>0$

\subsection{Evaluation criterion}

The objective function consists in minimizing the number of holes. The following function $F_{N H}$ counts the number of holes in image $A$ :

$$
F_{N H}(A)=\sum_{i} \sum_{j}\left(1-a_{i, j}\right) * a_{i-1, j} * a_{i, j-1} * a_{i+1, j} * a_{i, j+1}
$$

\subsection{Neighborhood structure}

An admissible solution is a binary image satisfying the orthogonal projections $(H, V)$ i.e, we relax the hole constraint in the definition of feasible solutions. We define the neighborhood of a solution as the set of images obtained by a single switching operation. Recall that a switching operation preserves the orthogonal projections. Fig. 4 illustrates an example of the switching operation.

This definition of neighborhood induces a graph $G(N, E)$ where the nodes of $G$ correspond to the binary images of $B M(H, V)$ and the edge set $E$ represents the neighborhood relation: $(A, B) \in E$ if and only if the binary images $A$ and $B$ are neighbors. We develop the following algorithm to select the best neighbor of the current solution according to the objective function: 
Input: $\{S$ a current solution $(S \in B M(H, V))\}$.

Output: $\left\{S^{\prime}\right.$ a best neighbor binary image of $\left.S\left(S^{\prime} \in B M(H, V)\right)\right\}$.

Repeat

Select a switching structure: $\{(i, j),(i, k),(h, j),(h, k)\}$

Apply switching operation;

Evaluate $S^{\prime}$

If $\left(F_{N H}\left(S^{\prime}\right) \leq F_{N H}(S)\right)$

Update the best neighbor obtained till now;

End If

Until no switches can be found;

\begin{tabular}{|c|c|c|c|c|c|c|c|c|c|}
\hline \multicolumn{5}{|c|}{$\begin{array}{c}\text { before switching operation } \\
j \quad k\end{array}$} & \multicolumn{5}{|c|}{$\begin{array}{c}\text { after switching operation } \\
j \quad k\end{array}$} \\
\hline & 1 & 1 & 0 & 0 & & 1 & 1 & 0 & 0 \\
\hline$i$ & 1 & 0 & 1 & 0 & $i$ & 1 & 1 & 0 & 0 \\
\hline$h$ & 0 & 1 & 0 & 1 & $h$ & 0 & 0 & 1 & 1 \\
\hline & 0 & 0 & 1 & 1 & & 0 & 0 & 1 & 1 \\
\hline
\end{tabular}

Figure 4. An example of the switching: $\{(i, j),(i, k),(h, j),(h, k)\}$.

\subsection{TS algorithm}

The parameters of the tabu search algorithm are defined as follows:

- Neighborhood move: The best neighbor binary image with the minimum holes which is tabu is selected as the new current solution. If all neighbors binary images are tabu, then the oldest binary image solution is selected as current solution.

- Tabu list and updating: The length of the tabu list is 5 binary images and tabu list is updated after each neighborhood switching operation.

- Termination criterion: When the best solution has not improved for the last 100 iterations or when the total number of iterations reaches 10000 .

The tabu search algorithm of reconstructing non hole binary images $\mathrm{RNH}(\mathrm{H}, \mathrm{V})$ is the following:

\section{Tabu search algorithm}

Input: \{binary image (Ryser solution)\}.

Output: $\{$ nearly non hole binary image respecting $H$ and $V$ \}.

While $(($ total $\leq 10000)$ and $(N b r \leq 100)$ do

Apply the procedure of the best neighbor solution;

Update the tabu list;

Update total and $\mathrm{Nbr}$;

End While 
The variable $\mathrm{Nbr}$ contains the number of iterations since the last improvement of the objective function. The variable total counts the number of iterations since the beginning of the algorithm.

\section{Numerical EXPERIMENTS}

We have implemented our algorithm in language $\mathrm{C}$, using the gcc compiler. All our experiments were run on an AMD Athlon XP-M $1.7 \mathrm{GHz}$ PC with $512 \mathrm{Mb}$ of memory.

In order to compare the relative efficiency of Ryser's algorithm and the tabu serach approach. for reconstructing matrices, we have used a large set of random binary images of various sizes. We generate a large set of square binary images with size varying from $40 \times 40$ to $200 \times 200$. For each size, we generate three classes of binary images $(A, B$, and $C)$. For each class $A, B$, and $C$, we generate binary images with $10 \%, 20 \%$, and $40 \%$ of black pixels, respectively. For each class, we take the average of 10 instances for each size. The results of computational experiments are summarized in Table 1 for class $A$, Table 2 for class $B$, and Table 3 for class $C$. In these tables, the sub-column (hole cell) contains the number of holes provided by each method. The sub-column labeled (Time) indicates the running time (in seconds) required by each method.

We note that the tabu search algorithm outperforms the classical approach of Ryser's even if the running time is slightly longer. In fact Ryser's algorithm can be regarded as a single iteration tabu search.

Table 1. Reconstruction results for TS algorithm (Class $A$ ).

\begin{tabular}{ccccc}
\hline \multirow{2}{*}{ Image } & \multicolumn{2}{c}{ Ryser Solution } & \multicolumn{2}{c}{ T.S. Solution } \\
\cline { 2 - 5 } & $\%$ hole & Time & $\%$ hole & Time \\
\hline $40 \times 40$ & $2.01 \%$ & 0.00 & $0.00 \%$ & 05.91 \\
\hline $60 \times 60$ & $2.47 \%$ & 0.01 & $0.00 \%$ & 05.86 \\
\hline $80 \times 80$ & $2.23 \%$ & 0.01 & $0.00 \%$ & 06.67 \\
\hline $100 \times 100$ & $1.95 \%$ & 0.01 & $0.00 \%$ & 06.95 \\
\hline $120 \times 120$ & $1.95 \%$ & 0.15 & $0.00 \%$ & 07.21 \\
\hline $140 \times 140$ & $2.12 \%$ & 0.15 & $0.01 \%$ & 08.00 \\
\hline $160 \times 160$ & $2.22 \%$ & 0.15 & $0.01 \%$ & 08.52 \\
\hline $200 \times 200$ & $2.13 \%$ & 0.31 & $0.01 \%$ & 09.01 \\
\hline
\end{tabular}


Table 2. Reconstruction results for TS algorithm (Class B).

\begin{tabular}{ccccc}
\hline \multirow{2}{*}{ Image } & \multicolumn{2}{c}{ Ryser Solution } & \multicolumn{2}{c}{ T.S. Solution } \\
\cline { 2 - 5 } & $\%$ hole & Time & $\%$ hole & Time \\
\hline $40 \times 40$ & $8.00 \%$ & 0.00 & $1.02 \%$ & 06.11 \\
\hline $60 \times 60$ & $9.88 \%$ & 0.01 & $0.97 \%$ & 06.26 \\
\hline $80 \times 80$ & $8.93 \%$ & 0.01 & $0.87 \%$ & 08.35 \\
\hline $100 \times 100$ & $7.80 \%$ & 0.02 & $0.78 \%$ & 09.18 \\
\hline $120 \times 120$ & $7.80 \%$ & 0.16 & $0.77 \%$ & 11.99 \\
\hline $140 \times 140$ & $8.51 \%$ & 0.15 & $0.85 \%$ & 12.57 \\
\hline $160 \times 160$ & $6.22 \%$ & 0.15 & $0.98 \%$ & 14.04 \\
\hline $180 \times 180$ & $6.07 \%$ & 0.22 & $0.81 \%$ & 14.44 \\
\hline $200 \times 200$ & $6.13 \%$ & 0.33 & $0.77 \%$ & 18.32 \\
\hline
\end{tabular}

Table 3. Reconstruction results for TS algorithm (Class $C$ ).

\begin{tabular}{ccccc}
\hline \multirow{2}{*}{ Image } & \multicolumn{2}{c}{ Ryser Solution } & \multicolumn{2}{c}{ T.S. Solution } \\
\cline { 2 - 5 } & $\%$ hole & Time & $\%$ hole & Time \\
\hline $40 \times 40$ & $16.00 \%$ & 0.00 & $1.81 \%$ & 07.01 \\
\hline $60 \times 60$ & $19.77 \%$ & 0.00 & $1.97 \%$ & 08.41 \\
\hline $80 \times 80$ & $17.87 \%$ & 0.02 & $1.37 \%$ & 08.82 \\
\hline $100 \times 100$ & $15.60 \%$ & 0.01 & $1.50 \%$ & 10.44 \\
\hline $120 \times 120$ & $15.61 \%$ & 0.16 & $1.55 \%$ & 12.27 \\
\hline $140 \times 140$ & $17.02 \%$ & 0.15 & $1.69 \%$ & 13.86 \\
\hline $160 \times 160$ & $17.62 \%$ & 0.15 & $2.00 \%$ & 16.93 \\
\hline $180 \times 180$ & $16.61 \%$ & 0.21 & $1.83 \%$ & 20.81 \\
\hline $200 \times 200$ & $17.04 \%$ & 0.32 & $2.04 \%$ & 21.32 \\
\hline
\end{tabular}

\section{Conclusion}

We have proposed a tabu-search-based approach to reconstructing binary images without interior hole pixels from its horizontal and vertical projections. The minimization of hole black pixels has been used as the objective function. The results obtained by this metaheuristic are encouraging. Our methodology can easily be applied to other types of hole pixels.

\section{REFERENCES}

[1] P. Balázs, A decomposition technique for reconstructing discrete sets from four projections, Image Vision Comput. 25 (2007), 1609-1619.

[2] P. Balázs, Reconstruction of Discrete Sets from Four Projections: Strong Decomposability, Electron. Notes Discrete Math. 20 (2005), 329-345.

[3] E. Barcucci, A. Del Lungo, M. Nivat, and R. Pinzani, Reconstructing convex polyominoes from their horizontal and vertical projections, Theor. Comput. Sci. 155 (1996), 321-347.

[4] J. Baumann, Z. Kiss, S. Krimmel, A. Kauba, A. Nagy, L. Rodek, S. Schillinger and J. Stephan, Discrete tomography methods for nondestructive testing, in: G. T. Herman 
and A. Kuba (eds.), Advances in Discrete Tomography and its Applications, Birkhäuser, Boston, 2007, 303-331.

[5] S. Brunetti, M. C. Costa, A. Frosini, F. Jarray and C. Picouleau, Reconstruction of binary matrices under adjacency constraints, in: G. T. Herman and A. Kuba (eds.), Advances in Discrete Tomography and its Applications, Birkhäuser, Boston, 2007, 125-150.

[6] S. Brunetti and A. Daurat, An algorithm reconstructing convex lattice sets, Theor. Comput. Sci. 304 (2003), 35-57.

[7] S. Brunetti, A. Del Lungo, F. Del Ristoro, A. Kuba and M. Nivat, Reconstruction of 4- and 8-connected convex discrete sets from row and column projections, Linear Algebra Appl. 339 (2001), 37-57.

[8] M. Chrobak and C. Dürr, Reconstructing hv-convex polyominoes from orthogonal projections, Inf. Process. Lett. 69 (1999), 283-289.

[9] M. C. Costa, F. Jarray and C. Picouleau, An acyclic days-off scheduling problem, 4ORQ. J. Oper. Res. 4 (2006), 73-85.

[10] M. C. Costa, F. Jarray and C. Picouleau, Reconstructing an alternate periodical binary matrix from its orthogonal projections, in: M. Coppo, E. Lodi and G. M. Pinna, Theoretical Computer Science, Lecture Notes in Computer Science 3701, Springer, 2005, 173-181.

[11] A. Del Lungo, A. Frosini, M. Nivat and L. Vuillon, Discrete tomography: Reconstruction under periodicity constraints, in: P. Widmayer et al (eds.), Automata, Languages and Programming, Lecture Notes in Computer Science 2380, Springer, 2002, 38-56.

[12] A. Del Lungo and M. Nivat, Reconstruction of connected sets from two projections, in: G. T. Herman and A. Kuba (eds.), Discrete Tomography: Foundations, Algorithms and Applications, Birkhäuser, Boston, 1999, 163-188.

[13] P. Hall, A model for learning human vascular anatomy, in: D.-Z. Du, P. M. Pardalos and J. Wang (eds.), DIMACS, Series in Discrete Mathematical Problems with Medical Applications 55, AMS, 2000, 11-27.

[14] A. Kuba and E. Balogh, Reconstruction of convex 2D discrete sets in polynomial time, Theor. Comput. Sci. 283 (2000), 223-242.

[15] R. W. Irving and M. R. Jerrum, Three-dimensional data security problems, SIAM J. Comput. 23 (1994), 170-184.

[16] F. Jarray, Solving Problems of Discrete Tomography. Applications in Workforce Scheduling, Ph.D. Thesis, University of CNAM, Paris, 2004.

[17] D. G. W. Onnasch and G. P. M. Prause, Heart chamber reconstruction from biplane angiography, in: G. T. Herman and A. Kuba (eds.), Discrete Tomography: Foundations, Algorithms and Applications, Birkhäuser, Boston, 1999, 385-403.

[18] H. R. Ryser, Combinatorial properties of matrices of zeros and ones, Canad. J. Math. 9 (1957), 371-377.

[19] B. Wang and F. Zhang, On the precise number of $(0,1)$-matrices in $U(r, s)$, Discrete Math. 187 (1998), 211-220.

[20] G. J. Woeginger, The reconstruction of polyominoes from their orthogonal projections, Inf. Process. Lett. 77 (2001), 225-229.

Alain Billionnet, Laboratoire Cedric-CNAM, 292 rue St-Martin, Paris, France e-mail: alain.billionnet@ensiie.fr

Fethi Jarray, Higher Institute of Computer Science of Medenine, Tunisia e-mail: fethi_jarray@yahoo.fr

Ghassen Tlig, Laboratoire LIMTIC, Institut Supérieur d'Informatqiue, Université de Tunis El Manar and Higher Institute of Computer Science of Mednine, Tunisia e-mail: ghassen.tlik@gmail.com

Ezzedine Zagrouba, Laboratoire LIMTIC, Institut Supérieur d'Informatqiue, Université de Tunis El Manar, Tunisia e-mail: ezzeddine.zagrouba@fsm.rnu.tn 